\title{
Novel variant in the FGD1 gene causing Aarskog-Scott syndrome
}

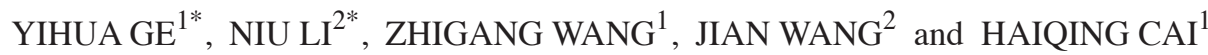 \\ ${ }^{1}$ Department of Pediatric Orthopedics; ${ }^{2}$ Institute of Pediatric Translational Medicine, \\ Shanghai Children's Medical Center, Shanghai Jiaotong University School of Medicine, Shanghai 200127, P.R. China
}

Received September 5, 2015; Accepted October 11, 2016

DOI: $10.3892 /$ etm.2017.4301

\begin{abstract}
Aarskog-Scott syndrome (ASS) is a rare, X-linked recessive inherited disorder. Affected individuals may develop short stature and exhibit distinctive skeletal and genital development. Mutations in the FYVE, rhogef and pleckstrin homology domain-containing protein 1 (FGDl) gene, located within the Xp11.21 region, are responsible for the occurrence of ASS. Since it is rare and complex, it can take a long time to obtain a definitive clinical diagnosis unless clinicians are familiar with the disease. In the present study, whole-exome sequencing (WES) was performed to screen for causal variants in a Chinese pediatric patient who exhibited a number of clinical symptoms of ASS, including short stature, facial abnormalities, stubby metacarpals and swollen testis. DNA sequencing revealed a novel c.1270 A>G mutation in exon 6 of the FGDl gene, which led to an amino acid conversion of asparagine to aspartic acid on codon 424 and in silico analysis indicated that this novel missense mutation was pathogenic. The present study identified a novel variant of the FGDl gene and to the best of our knowledge, is the first report of ASS in a Chinese individual. The results indicated that WES is an effective tool for the diagnosis of rare and complex syndromes such as ASS.
\end{abstract}

\section{Introduction}

Aarskog-Scott syndrome (ASS), also known as faciogenital dysplasia [Online Mendelian Inheritance in Man (OMIM) ID no. 305,400, http://www.omim.org/entry/305400], is a rare, $\mathrm{X}$-linked recessive inherited disease caused by a mutation in the FYVE, rhogef, and pleckstrin homology domain-containing protein 1 (FGDI) gene (1). Affected

Correspondence to: Dr Haiqing Cai, Department of Pediatric Orthopedics, Shanghai Children's Medical Center, Shanghai Jiaotong University School of Medicine, 1678 Dongfang Road, Shanghai 200127, P.R. China

E-mail: caihaiqing@scmc.com.cn

${ }^{*}$ Contributed equally

Key words: Aarskog-Scott syndrome, whole-exome sequencing, FGD1 gene, novel variant, rare complex syndrome subjects may develop short stature, facial abnormalities (hypertelorism, long philtrum, abnormal auricles), skeletal anomalies (short limbs, broad hands and feet) and genital hypoplasia (shawl scrotum) (1-3). Specific cases may present with unusual manifestations, which makes diagnosis difficult and the clinical features of ASS are easily confused with those of a number of other complex syndromes, particularly prior to obtainment of definitive biochemical findings (1). These include Noonan's syndrome (OMIM ID. 163,950, http://www. omim.org/entry/163950) and Robinow's syndrome (OMIM ID. 180,700, http://www.omim.org/entry/180700) (4). It can take a long time to obtain a definite clinical diagnosis of ASS due to its complexity and rare nature. For this reason, it is necessary to establish a prompt and accurate diagnostic method. Whole-exome sequencing (WES) is an appropriate detection technology due to its high throughput screening and ability to identify causal gene variants.

The FGDl gene is located in the Xp11.21 region, containing 18 exons and encoding for 961 amino acids (5). The FGDl gene encodes a protein that contains a RhoGEF homology domain, two pleckstrin homology domains and a FYVE-zinc finger domain (6). Each domain contains a different N-terminal domain, indicating regulation with distinct sets of downstream target genes. The encoded protein specifically binds to the Rho family GTPase cell division control protein 42 homolog (Cdc42) and can stimulate the guanosine diphosphate to guanosine triphosphate (GDP-GTP) exchange of the isoprenylated form of Cdc42 (5-7). Defects in the FGDl gene may therefore be responsible for the onset of ASS.

In the present study, genetic analysis using WES technology was performed on one Chinese pediatric patient who presented with clinical manifestations of ASS, including short stature, facial abnormalities, stubby metacarpals and swollen testis. The patient was eventually diagnosed with ASS and one novel variant was located in the FGDl gene, using DNA sequencing technology.

\section{Patients and methods}

Clinical presentation of the patient. A boy, 2 years and 2 months of age, was admitted to Shanghai Children's Medical Center (Shanghai, China) in December 2014 due to exhibiting short stature and facial abnormalities. The patient had been a full-term baby and the delivery had been uneventful. The patient had a birth weight of $3.15 \mathrm{~kg}$ and was $50 \mathrm{~cm}$ in body length at birth. The patient's parents were physically healthy, 
and the marriage was non-consanguineous. The patient's father was $168 \mathrm{~cm}$ in height and the mother was $153 \mathrm{~cm}$. The patient began speaking at the age of 12 months and walking at the age of 15 months. Physical examination indicated a body weight of $10 \mathrm{~kg}$, a height of $79.5 \mathrm{~cm}$ and a head circumference of $47 \mathrm{~cm}$. The patient had facial abnormalities, including orbital hypertelorism, blepharoptosis, low ears and a groove below the upper lip, and the eyes appeared dull (Fig. 1A). X-ray results revealed that the metacarpals were visibly stubby (Fig. 1B). The patient had male-appearing genitalia, but the bilateral testicles were swollen, and an inguinal hernia was observed.

The X-ray of the hand suggested that his bone age was 15 months (Fig. 1B). The ultrasound results indicated the presence of a small $6 \times 5-\mathrm{mm}$ cyst on the spleen and a slightly greater than average liver size (the diagonal diameter of the right lobe of the liver of the patient was $96 \mathrm{~mm}$; normal range, 85-92 $\mathrm{mm}$ ). The blood tests for the functions of thyroid, liver, kidney, blood glucose and electrolytes were normal (data not included). The subject was considered to have dyschondroplasia caused by a single gene mutation. In order to produce a clear clinical diagnosis quickly, the mutant genes were screened using WES. The present study was approved by the ethics committee of the Shanghai Children's Medical Center, Shanghai Jiaotong University School of Medicine. Informed consent (including publication of the genetic data and images) was obtained from the patient's parents prior to screening.

WES and data analysis. The genomic DNA of the patient and parents was isolated from peripheral blood samples using a QIAamp DNA Blood Mini kit ${ }^{\circledR}$ (Qiagen GmbH, Hilden, Germany). A total of $3 \mu \mathrm{g}$ DNA from the patient was sheared to produce 150-200 base pair (bp) fragments. An adapter-ligated library was prepared using an Agilent SureSelect Target Enrichment system (Agilent Technologies, Inc., Santa Clara, CA, USA) and enrichment of coding exons and flanking intronic regions was performed using Agilent SureSelect Human All Exon V6 reagent (Agilent Technologies, Inc.), according to the manufacturer's protocol. Clusters were subsequently generated using isothermal bridge amplification with an Illumina cBot system (Illumina Inc., San Diego, CA, USA) and sequencing was performed using an Illumina HiSeq 2000 system (Illumina, Inc.).

Base calling and assessment of sequence read quality was performed using Illumina Sequence Control Software (SCS; Illumina, Inc.) with real-time analysis. Alignment of sequence reads to a reference human genome (Human 37.3, single nucleotide polymorphism 135) was performed using NextGENe ${ }^{\circledR}$ (SoftGenetics, LLC., State College, PA, USA). All single-nucleotide variants (SNVs) and indels were saved in a variant call format and uploaded for Ingenuity ${ }^{\circledR}$ Variant Analysis $^{\mathrm{TM}}$ (Ingenuity Systems; Qiagen $\mathrm{GmbH}$ ) for biological analysis and interpretation.

Sanger sequencing verification of the FGDl gene. The primers for the amplification of FGDl (GenBank accession no. NM_004463.2) were designed using UCSC ExonPrimer online software (http://genome.ucsc.edu/index.html). The primers designed for exon 6 of $F G D 1$ gene were as follows: Forward, 5'-AАCCCTAАCCCCATTCCCTG-3' and reverse, 5'-GCCCGGCTCTGATTCCTTTT-3'. Both exons and
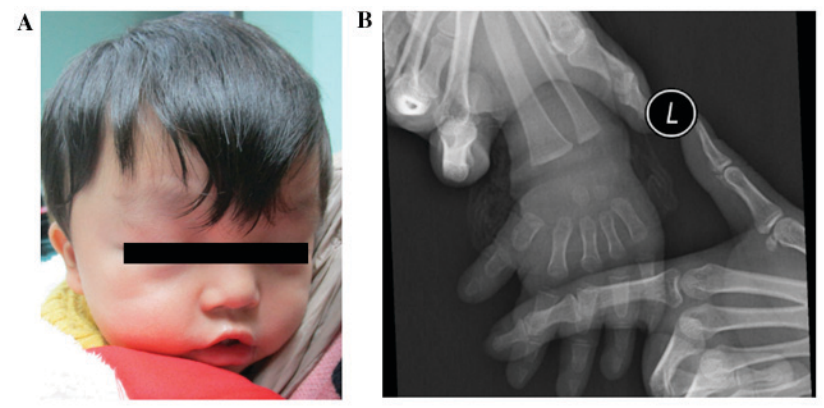

Figure 1. Clinical abnormalities of the patient. (A) Image of the patient Facial abnormalities included orbital hypertelorism, blepharoptosis, low ears and a groove below the upper lip, and the eyes appeared dull. (B) X-ray of the patient's hand (held in position by a parent) revealing visibly stubby metacarpals. 'L' indicates the left hand.

exon-intron boundaries were amplified using polymerase chain reaction (PCR). The reaction mixture for each amplification contained 1X Premix Taq (Ex Taq version 2.0; RR003; Takara Biotechnology Co., Ltd., Dalian, China), and reaction was carried out with the following PCR conditions: Initial denaturation at $95^{\circ} \mathrm{C}$ for $5 \mathrm{~min}$, then 19 cycles of $95^{\circ} \mathrm{C}$ for $30 \mathrm{sec}$, $65^{\circ} \mathrm{C}$ for $30 \mathrm{sec}$ and $72^{\circ} \mathrm{C}$ for $45 \mathrm{sec}$, followed by 14 cycles of $95^{\circ} \mathrm{C}$ for $30 \mathrm{sec}, 55^{\circ} \mathrm{C}$ for $30 \mathrm{sec}$ and $72^{\circ} \mathrm{C}$ for $45 \mathrm{sec}$, and a final elongation step at $72^{\circ} \mathrm{C}$ for 5 min using a $\mathrm{C} 1000^{\mathrm{TM}}$ Thermal Cycler PCR instrument (Bio-Rad Laboratories, Inc., Hercules, CA, USA). The products were examined on a $1 \%$ agarose gel and purified with a QIAquick Gel Extraction kit (Qiagen $\mathrm{GmbH}$ ). The resulting DNA was sequenced using the ABI3730XL sequencer (Applied Biosystems; Thermo Fisher Scientific, Inc., Waltham, MA, USA) with the forward and reverse primers. The sequence data was then analyzed using Mutation Surveyor ${ }^{\circledR}$ DNA Variant Analysis Software (version 4.0.4; SoftGenetics, LLC.).

In silico analysis of the function of the identified variant. The function of the identified variant was analyzed with MultAlin online software (http://multalin.toulouse.inra.fr/multalin/), SIFT online software (http://sift.jcvi.org/) and PolyPhen-2 online software (http://genetics.bwh.harvard.edu/pph2/). The three-dimensional structure of the wild-type FGD1 protein was obtained from the Protein Data Bank (http://www.rcsb. org/pdb/home/home.do), and was introduced in the Pymol software (version 1.8.4.0; https://www.pymol.org; Schrödinger, Inc., New York, NY, USA). The three-dimensional structure of the mutant FGD1 protein was produced after changing asparagine to aspartic acid on codon 424.

\section{Results}

Identification and confirmation of the causal variant. To obtain a clear clinical diagnosis quickly, the patient was screened for causal variants using WES. The candidate variants were first screened with the criterion of a minor allele frequency $<3 \%$ against the 1,000 Genomes Project, the NHLBI exome variant server or in 50 HapMap control exomes. The area of analysis included each exon and 20 bp of exon-intron boundaries. Subsequently, clinical symptoms of short stature, facial abnormalities and stubby metacarpals were selected as 
$\mathbf{A}$

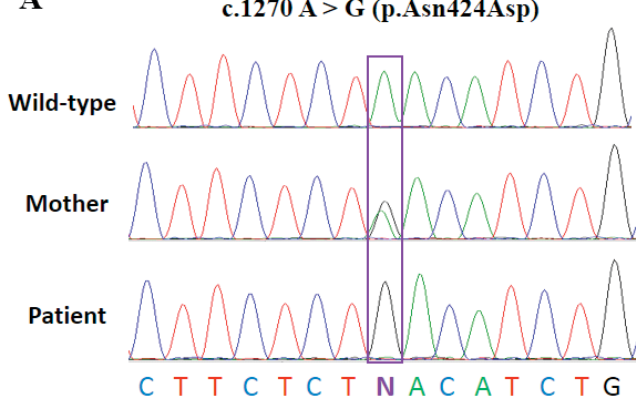

B $\begin{array}{cccc}410 & 420 & 430 & 440 \\ \text { Homo sapiens EEARNRSEPADVVHGIFSNICSIYCFHQOFLLPELE }\end{array}$ Homo sapiens EEARNRSSFPADVVHGIFSNICSIYCFHQQFLLPELEKRM
Macaca mulatta EEARNRSSFPADVVHGIFSNICSIYCFHOQFLLPETEKRM Mus musculus FFARNRSSFPADVVHGIFSNICS TYCFHOOFLTPEIFKRM Bos Taurus EEARNRSS F PADVVHG IF SNICS IYCFHOOFLLPELEKRM Rattus norvegicus EEARNRS SFPADVVHGIFSNICS I YCFHQQFLLPELEXRM Felis catus EEARNRS SEPADVVHGIFSNICS I YCFHQQFLLPELEKRM Canis lupus EEARNRSSEPADVVHGIFSNICS IYCFHQQFLLPELEKRM

Figure 2. Verification and function prediction of the c.1270 A>G variant in the FGD1 gene. (A) Sanger sequencing results indicated that the patient was hemizygotic for c. $1270 \mathrm{~A}>\mathrm{G}$, and a heterozygote of the locus was detected in the patient's mother. (B) The referred amino acid of codon 424 is highly evolutionarily conserved. FGD1, FYVE, RhoGEF and pleckstrin homology domain-containing 1; A, alanine; G, glycine.

the filtering indices to analyze the screened candidate variants. Ultimately, a novel hemizygous variant (c.1270 A>G) in the FGDl gene was identified and was found to contribute to the patient's condition. Results from sequencing analysis indicated that the c.1270 $\mathrm{A}>\mathrm{G}$ mutation at codon 424 of exon 6 was a missense mutation that led to an amino acid conversion of asparagine to aspartic acid on codon 424 (p.Asn424Asp) and Sanger sequencing confirmed the presence of this variant in the patient (Fig. 2A). In addition, direct sequencing results revealed the patient's mother to be heterozygous for the same variant (Fig. 2A), which is consistent with the mode of inheritance of the FGDl gene. This suggested that the identified variant in the FGDl gene was the genetic cause of the patient's condition.

Analysis of the function of the identified variant. To evaluate the pathogenicity of the variant in the FGDl gene, a number of in silico tools were used to analyze it. Results of analysis using MultAlin software indicated that the amino acid asparagine (Asn) was highly evolutionarily conserved at codon 424 (Fig. 2B). The protein variant was predicted to be damaged by both PolyPhen-2 and SIFT software. These findings suggested that the c.1270 A>G variant in the FGD1 gene causes ASS. In order to improve the understanding of the variant of the FGDl gene, a three-dimensional model of the FGD1 protein was built (Fig. 3). Under normal circumstances, a hydrogen bond forms between the nitrogen atom of Asn (p.424) and the oxygen atom of Asn (p.473), and the hydrogen bond is located in the key curve site in the secondary structure of the protein. The p.Asn424Asp variant is indicated to disrupt the formation of the hydrogen bond and therefore disrupts the normal protein structure.

\section{Discussion}

Ever since ASS was identified by Aarskog in 1970 and later by Scott in $1971(8,9)$, more than 60 cases have been described worldwide (10). However, only 37 cases have been confirmed using molecular testing, which are included in the Human Gene Mutation Database (http://www.hgmd.cf.ac.uk/ac/). Furthermore, to the best of our knowledge, no case of a Chinese patient with ASS has been previously reported. Table I presents the clinical manifestations of ASS in patients carrying the FGDl mutation in different exons/introns, using information

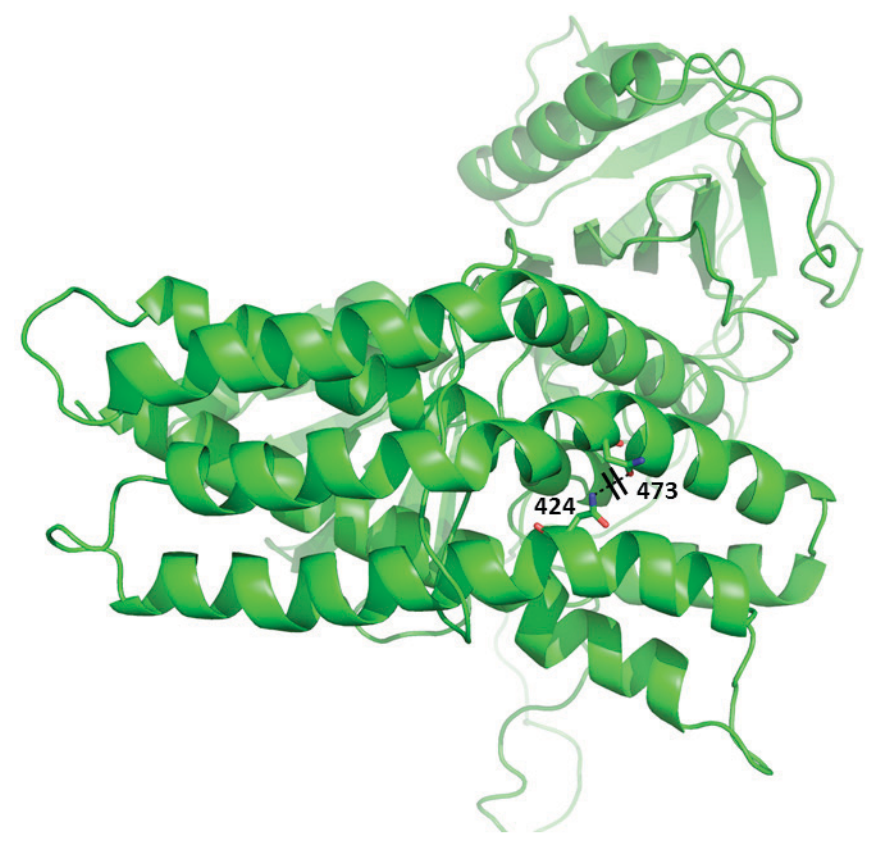

Figure 3. Three-dimensional structure model of the FGD1 protein. The nitrogen atom of Asn (p.424) connects to the oxygen atom of the Asn (p.473) via a hydrogen bond. Nitrogen atoms are show in blue, oxygen atoms in red and a broken hydrogen bond by a double black slash. FGD1, FYVE, RhoGEF and pleckstrin homology domain-containing 1; Asn, asparagine.

from previous studies $(1,4,6,11,12)$. This may facilitate the direct analysis of genotype-phenotype correlations. Typically, the clinical features of ASS include facial abnormities, short stature, distinctive skeletal and genital developmental abnormalities and numerous other uncommon features that have been described in the literature, such as mental retardation $(2,3,13,14)$. The clinical features exhibited by the patient in the present study were consistent with this. In addition to the aforementioned clinical manifestations, the patient exhibited various degrees of abnormalities of the liver and spleen, which have not been reported in previous cases.

It is not easy to distinguish ASS from Noonan's syndrome and Robinow's syndrome on the basis of clinical manifestations alone. This indicates that conventional direct sequencing is unsuitable for the molecular diagnosis of ASS. Furthermore, direct sequencing has certain technical 


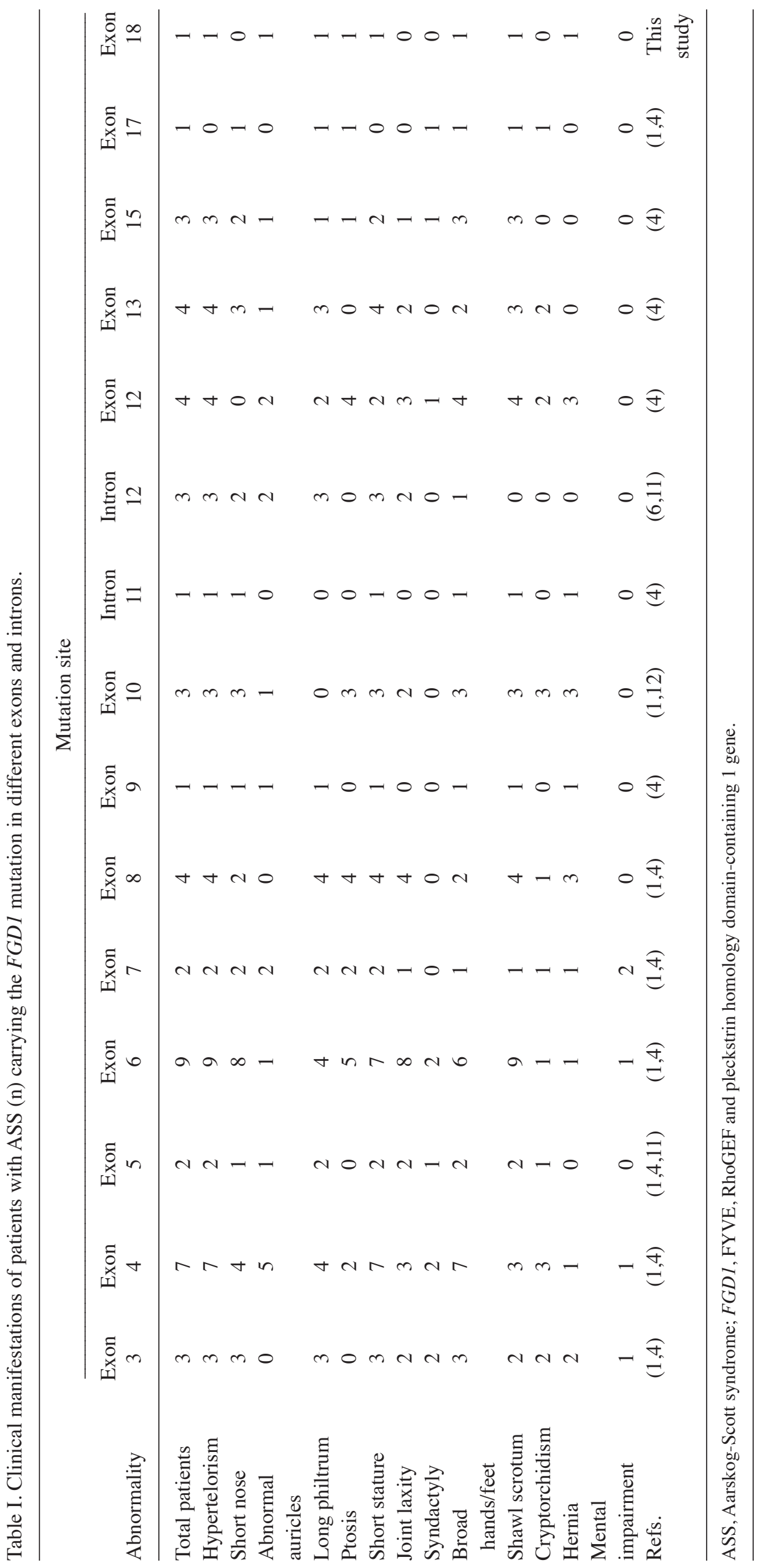


limitations, particularly in the detection of gross deletions and duplications, and the rate of detection of mutations is low (15). With the advent of next-generation sequencing (NGS), the diagnostic efficiency of complex syndromes has increased considerably. WES is one type of NGS and has been successfully applied in the diagnosis of ASS to identify a branch point variant in the FGDl gene that conventional Sanger sequencing failed to detect (6). Another important traditional genetic method of testing for ASS is multiplex ligation-dependent probe amplification (MLPA). It is used to detect deletions and duplications of several exons, and has successfully detected a case (16). Copy number variant (CNV) sequencing technology based on WES has been designed to add probes for calling copy number in the intron area. That means that it is able to identify SNVs, loss of heterozygosity and CNVs. Thus, it has the potential to replace Sanger sequencing, MLPA and gene chip technologies as a diagnostic tool $(17,18)$. High-throughput sequencing may be the first-tier test for complex syndromes in the future.

To the best of our knowledge, the FGDl gene is the only known causative gene for ASS (1). A total of 61 variants have been identified in the FGDl gene, including 32 missense variants, 6 nonsense variants, 16 frameshift variants, 4 splice site variants, 1 in-frame deletion and 2 gross deletions (19). In the present study, a novel hemizygous variant of the FGDl gene was successfully identified in a Chinese patient with ASS. Sequence analysis indicated that the c.1270 A>G mutation in exon 6 could result in an amino acid conversion (p.Asn424Asp). The mother of the patient was heterozygous for the same variant. The mutation was novel in that the allele frequency of this variant was absent from the 1,000 Genomes Project and the Exome Aggregation Consortium databases and to the best of our knowledge, it is the first report of a mutation at the c.1270 site. A variety of in silico studies were performed to evaluate the pathogenicity of the variant. Results analyzed by MultAlin software demonstrated that the Asn in codon 424 was highly conserved, indicating a crucial function of the amino acid. Results of PolyPhen-2 and SIFT online prediction software packages suggested that the identified variant impairs FGD1 protein synthesis, leading to a deleterious mutation, which means that it is a disease-causing variant. In addition, the 3D structure of the FGD1 protein suggested that the mutation may break the hydrogen bond formed between the nitrogen atom of Asn (p.424) and the oxygen atom of Asn (p.473), which may make the micro-curve disappear. The alteration in the secondary structure changes the normal structure of the RhoGEF homology domain (residues 373-561) and ultimately affects the function of the FGD1 protein.

In conclusion, in the present study, a hemizygous variant in the FGDl gene was identified in a Chinese pediatric patient with ASS. The c.1270 A>G mutation is a missense mutation that caused an amino acid conversion (p.Asn424Asp). The allele frequency of this variant has not been detected previously; therefore, a preliminary analysis of the molecular basis of the novel variant linked to the occurrence of ASS was performed. PolyPhen-2 and SIFT software analysis suggested that this variant was associated with protein damage, which is consistent with results of an analysis of the 3D structure. Furthermore, genotype-phenotype correlation studies provided evidence to support the pathogenicity of this variant. According to the latest criteria of the American College of Medical Genetics and Genomics (20), the c.1270 A>G (p.Asn424Asp) mutation was classified as likely pathogenic. However, the precise molecular mechanisms of the differences in the manifestation of ASS remain undefined and require further study.

\section{Acknowledgements}

The current study was supported by the National Natural Science Foundation of China (grant nos. 81201353 and 81472051). The authors are deeply grateful to the patient and his family for participating in the present study.

\section{References}

1. Orrico A, Galli L, Cavaliere ML, Garavelli L, Fryns JP, Crushell E, Rinaldi MM, Medeira A and Sorrentino V: Phenotypic and molecular characterisation of the Aarskog-Scott syndrome: A survey of the clinical variability in light of FGD1 mutation analysis in 46 patients. Eur J Hum Genet 12: 16-23, 2004.

2. Orrico A, Galli L, Buoni S, Hayek G, Luchetti A, Lorenzini S, Zappella M, Pomponi MG and Sorrentino V: Attention-deficit/hyperactivity disorder (ADHD) and variable clinical expression of Aarskog-Scott syndrome due to a novel FGD1 gene mutation (R408Q). Am J Med Genet A 135: 99-102, 2005.

3. Kaname $\mathrm{T}$, Yanagi $\mathrm{K}$, Okamoto $\mathrm{N}$ and Naritomi $\mathrm{K}$ : Neurobehavioral disorders in patients with Aarskog-Scott syndrome affected by novel FGD1 mutations. Am J Med Genet A 140: 1331-1332, 2006.

4. Völter C, Martínez R, Hagen R and Kress W: Aarskog-Scott syndrome: A novel mutation in the FGD1 gene associated with severe craniofacial dysplasia. Eur J Pediatr 173: 1373-1376, 2014.

5. Pasteris NG, Buckler J, Cadle AB and Gorski JL: Genomic organization of the faciogenital dysplasia (FGD1; Aarskog syndrome) gene. Genomics 43: 390-394, 1997.

6. Aten E, Sun Y, Almomani R, Santen GW, Messemaker T, Maas SM, Breuning MH and den Dunnen JT: Exome sequencing identifies a branch point variant in Aarskog-Scott syndrome. Hum Mutat 34: 430-434, 2013.

7. Hou P, Estrada L, Kinley AW, Parsons JT, Vojtek AB and Gorski JL: Fgd1, the Cdc42 GEF responsible for Faciogenital Dysplasia, directly interacts with cortactin and mAbp1 to modulate cell shape. Hum Mol Genet 12: 1981-1993, 2003.

8. Aarskog D: A familial syndrome of short stature associated with facial dysplasia and genital anomalies. J Pediatr 77: 856-861, 1970.

9. Scott CI: Unusual facies, joint hypermobility, genital anomaly and short stature: A new dysmorphic syndrome. Birth Defects Orig Artic Ser 7: 240-246, 1971.

10. Orrico A, Galli L, Faivre L, Clayton-Smith J, Azzarello-Burri SM, Hertz JM, Jacquemont S, Taurisano R, Arroyo Carrera I, Tarantino E, et al: Aarskog-Scott syndrome: Clinical update and report of nine novel mutations of the FGD1 gene. Am J Med Genet A 152A: 313-318, 2010.

11. Pérez-Coria M, Lugo-Trampe JJ, Zamudio-Osuna M, Rodríguez-Sánchez IP, Lugo-Trampe A, de la Fuente-Cortez B, Campos-Acevedo LD and Martínez-de-Villarreal LE: Identification of novel mutations in Mexican patients with Aarskog-Scott syndrome. Mol Genet Genomic Med 3: 197-202, 2015.

12. Orrico A, Galli L, Falciani M, Bracci M, Cavaliere ML, Rinaldi MM, Musacchio A and Sorrentino V: A mutation in the pleckstrin homology (PH) domain of the FGD1 gene in an Italian family with faciogenital dysplasia (Aarskog-Scott syndrome). FEBS Lett 478: 216-220, 2000.

13. Lebel RR, May M, Pouls S, Lubs HA, Stevenson RE and Schwartz CE: Non-syndromic X-linked mental retardation associated with a missense mutation (P312L) in the FGD1 gene. Clin Genet 61: 139-145, 2002.

14. Diluna ML, Amankulor NM, Johnson MH and Gunel M: Cerebrovascular disease associated with Aarskog-Scott syndrome. Neuroradiology 49: 457-461, 2007. 
15. Ng SB, Buckingham KJ, Lee C, Bigham AW, Tabor HK, Dent KM, Huff CD, Shannon PT, Jabs EW, Nickerson DA, et al Exome sequencing identifies the cause of a mendelian disorder. Nat Genet 42: 30-35, 2010.

16. Ronce N, Maystadt I, Hubert C, Vonwill S, Devriendt K, Moizard MP and Raynaud M: Aarskog-Scott syndrome: First report of a duplication in the FGD1 gene. Clin Genet 82: 93-96, 2012.

17. Zhao M, Wang Q, Wang Q, Jia P and Zhao Z: Computational tools for copy number variation (CNV) detection using next-generation sequencing data: Features and perspectives. BMC Bioinformatics 14 (Suppl 11): S1, 2013.

18. Janevski A, Varadan V, Kamalakaran S, Banerjee $\mathrm{N}$ and Dimitrova N: Effective normalization for copy number variation detection from whole genome sequencing. BMC Genomics 13 (Suppl 6): S16, 2012.
19. Orrico A, Galli L, Clayton-Smith J and Fryns JP: Clinical utility gene card for: Aarskog-Scott Syndrome (faciogenital dysplasia)update 2015. Eur J Hum Genet 23, 2015.

20. Richards S, Aziz N, Bale S, Bick D, Das S, Gastier-Foster J, Grody WW, Hegde M, Lyon E, Spector E, et al: Standards and guidelines for the interpretation of sequence variants: A joint consensus recommendation of the American college of medical genetics and genomics and the association for molecular pathology. Genet Med 17: 405-424, 2015. 